\title{
The prospects for the study of the avirulence genes characteristic for the West Siberian population of wheat stem rust Puccinia graminis f. sp. tritici
}

\author{
Vasiliy Kelbin \\ ICG SB RAS, Novosibirsk, Russia \\ kelbin@bionet.nsc.ru
}

\author{
Ekaterina Sergeeva \\ ICG SB RAS, Novosibirsk, Russia \\ sergeeva@bionet.nsc.ru \\ Elena Salina \\ Kurchatov Genomics Center, \\ ICG SB RAS, Novosibirsk, Russia \\ salina@bionet.nsc.ru
}

\author{
Ekaterina Skolotneva \\ ICG SB RAS, Novosibirsk, Russia \\ skolotnevaes@bionet.nsc.ru
}

\begin{abstract}
Wheat stem rust is a plant disease caused by pathogenic fungus Puccinia graminis f. sp. tritici which leads to significant damage of the crop. During the last decade the disease affected the crops in West Siberia. The plant response to infection is determined by the correspondence of host resistance $(\mathrm{Sr})$ and fungus avirulence/virulence $(\mathrm{Avr} / v r)$ genes. We first characterized the West Siberian population of wheat stem rust: the probable sources of infection were traced, the race composition and main avirulence genes were defined. Four fungal lines with contrasting patterns of $A v r$ genes were selected for whole genomic sequencing.
\end{abstract}

Keywords - bread wheat, stem rust, Avr gene, Sr gene, genome sequencing

\section{Motivation}

The interaction of bread wheat Triticum aestivum L. $(2 \mathrm{n}=$ $6 \mathrm{x}=42$ ) and stem rust Puccinia graminis f. sp. (forma specialis) tritici is a promising model for the study of "plant host - pathogen" coevolution. This model can elaborate the genetic basis of plant immunity response and pathogenesis, and is also relevant for breeding requirements. During the last decade the extensive outbreaks of stem rust occured at the West Siberia region [1]. Plant breeding for immunity to stem rust requires the extensive data on genetic characteristics of pathogen population. Our study is dedicated to analysis of West Siberian local population of fungal pathogen $P$. graminis f. sp. tritici.

\section{Methods}

To describe the population we applied the methods of phytopathology and molecular genetics. Initially, we collected the samples of stem rust originated from Novosibirsk, Omsk and Altay regions during the wheat vegetations of 2017-2019 years. Totally we obtained the 94 single pustule isolates (genetically homogeneous lines) that are maintained in laboratory conditions.

\section{Results}

First, we identified the type of life cycle for the local (Novosibirsk) stem rust population. The fungus $P$. graminis can realize complete (sexual) or partial (asexual) life cycles. In case of complete cycle, the barberry (Berberis) plant is used as alternate host on which the sexual process is carried out [2]. We compared the genotyping profiles for 11 molecular SSRmarkers (simple sequence repeats) of 60 rust samples collected from wheat, barberry and wild grasses $[3,4,5]$. Processed using the PAST3 program, SSR profiles obtained from barberry and wild grasses were clustered in the same group and the samples from wheat to distinct one. Therefore, sexual life for $P$. graminis $\mathrm{f}$. sp. secalis through barberries and wild grasses and asexual life cycle for wheat stem rust P. graminis f. sp. tritici were supposed for local in the Novosibirsk region.

Then, we evaluated the avirulence genes (Avr) polymorphisms and the race composition of local population of stem rust. The infection types of single pustule isolates $P$. graminis f. sp. tritici were tested on special set of rust resistant/susceptible wheat lines (Pgt differential set, AAFC Cereal Research Centre, Canada). In the Novosibirsk region, we identified one predominant race TKRPF and the minor races differing for 1-3 genes. The cluster analysis (PAST3, Multivariate Software) of the samples originated from Novosibirsk, Omsk and Altay regions demonstrated that there are two independent pathogen subpopulations presented in Omsk and Altay regions sharing the races at the contact area of Novosibirsk region. Thus, wheat stem rust in the Novosibirsk region is not endemic but carried by wind from both neighboring regions. This is confirmed by the previous conclusion that barberry is not a source of infection for wheat crops in the Novosibirsk region.

We postulated the profiles of the avirulence/virulence $(A v r / v r)$ genes and their allelic states in local Novosibirsk population. The 51 single pustule isolates obtained in 2017 , 2018 were twice tested by virulence to wheat lines with $\mathrm{Sr}$ genes (Pgt differential set), and the data were processed with the PAST3 program. The most of $P$. graminis f. sp. tritici isolates were virulent to lines with resistance genes $\operatorname{Sr} 5, \operatorname{Sr} 9 a$, $\operatorname{Sr} 9 g, \operatorname{Sr} 10, \operatorname{Sr} 38, \operatorname{SrMcN}$, and avirulent to lines with $\operatorname{Sr} 24$, $\operatorname{Sr} 30, \operatorname{Sr} 31$. Also we found the changes in the frequencies of some reaction types on lines with $\operatorname{Sr} 6, \operatorname{Sr} 7 b, \operatorname{Sr} 8 a, \operatorname{Sr} 9 b, \operatorname{Sr} 9 d$, $\operatorname{Sr} 9 e, \operatorname{Sr} 11$, Sr17, Sr21, Sr36, and SrTmp genes that could be used for tracing the dynamics of virulent/avirulent types in Novosibirsk region. That way, we state the presence of the avirulence genes in local $P$. graminis f. sp. tritici population: AvrSr6, AvrSr7b, AvrSr8a, AvrSr9b, AvrSr9e, AvrSr11, AvrSr17, AvrSr21, AvrSr24, AvrSr30, AvrSr31, AvrSr36, AvrSrTmp.

As a result of the foregoing we characterized the West Siberia (in particular local Novosibirsk population) for the first time. The data obtained in the study let us select the four single pustule isolates with contrasting patterns of $A v r$ genes for further genomic analysis. We performed the whole genome Illumina sequencing for 4 samples in two replications 
each at the NextSeq550 capacity of Core Facility of Genomics Investigations (Institute of Cytology and Genetics SB RAS) and obtained from 14,8 to 26 millions of pair end reads $(2 \times 150$ $\mathrm{bp})$, that corresponds to coverage of $50 \mathrm{X}$ and higher. Although the reference sequence of $P$. graminis $\mathrm{f}$. sp. tritici is now publicly available, the studies of fungal $A v r$ genes structure are at the ongoing stage: proposed numerous candidated for pathogen effector genes [5, 6, 7]. The whole genomic sequencing of isolates (lines) with different $A v r$ spactra, further data processing (de novo assembling, reads mapping to reference sequence) and comparative genomic analysis will enable us to develop the molecular SNP (single nucleotide polymorphism) and SSR (simple sequence repeat) markers either for fast efficient pathogen monitoring at the local area or for using in study of interaction "host plant - pathogen" model.

\section{Acknowledgment}

The work was supported by RFBR 17-29-08018 and the budgetary project No. 0259-2019-0001-C-01.

\section{References}

[1] Skolotneva E.S., Salina E.A. (2016) Features of the causative agent of wheat stem rust in the conditions of Western Siberia. In: Protection of grain crops from diseases, pests, weeds: achievements and problems. Bolshiye Vyazemy. 67-71

[2] Leonard K.J., Szabo L.J. (2005) Stem rust of small grains and grasses caused by Puccinia graminis. Molecular plant pathology. 6(2): 99-111.

[3] Berlin A. et al. (2012) Genetic variation in Puccinia graminis collected from oats, rye, and barberry. Phytopathology. 102(10): 1006-1012.

[4] Zhong S. et al. (2009) Development and characterization of expressed sequence tag-derived microsatellite markers for the wheat stem rust fungus Puccinia graminis f. sp. tritici. Phytopathology. 99(3): 282-289.

[5] Ellis J.G. et al. (2007) Wheat rust resistance research at CSIRO. Australian Journal of Agricultural Research. 58(6): 507-511.

[6] Rutter W.B. et al. (2017) Divergent and convergent modes of interaction between wheat and Puccinia graminis f. sp. tritici isolates revealed by the comparative gene co-expression network and genome analyses. BMC genomics. 18(1): 291.

[7] Sperschneider J. et al. (2014) Diversifying selection in the wheat stem rust fungus acts predominantly on pathogen-associated gene families and reveals candidate effectors. Frontiers in plant science. 5: 372. 